\title{
Reconstruction from Digital Holograms by Statistical Methods
}

\author{
Saowapak Sotthivirat and Jeffrey A. Fessler \\ Dept. of Electrical Engineering and Computer Science \\ University of Michigan \\ Ann Arbor, MI 48109
}

\begin{abstract}
Conventional numerical reconstruction for digital holography using a filter applied in the spatial frequency domain to extract the primary image may yield suboptimal image quality because of the loss in highfrequency components and interference from other undesirable terms of a hologram. In this paper, we propose a new numerical reconstruction approach using a statistical technique. This approach reconstructs the complex field of the object from the real-valued hologram intensity data. Because holographic image reconstruction is an ill-posed problem, our statistical technique is based on penalized-likelihood estimation. We develop a Poisson statistical model for this problem and derive an optimization transfer algorithm that monotonically decreases the cost function each iteration. Simulation'results show that our statistical technique has the potential to improve image quality in digital holography relative to conventional reconstruction techniques.
\end{abstract}

\section{Introduction}

Digital recording of a hologram on a digital camera and a numerical reconstruction of a complex wavefield on a computer are attractive due to time-consuming processes of optical recording on photographic film and optical reconstruction preparation in conventional holography. In 'digital off-axis holography [1], the most common approach for extracting only the real or virtual image using numerical reconstruction is to perform a digital spatial filter on the hologram [2]. The main drawbacks of this approach are the loss of high frequency components in the reconstructed holographic image and interference from other terms in the hologram. Moreover, this approach is limited by a plane wave assumption of the reference beam. Phaseshifting $[3,4]$ and phase modulation $[5]$ methods were proposed to suppress the zero-order image and one of the twin images, but they require at least three holograms to reconstruct one holographic image. The approach proposed in [6] retrieves the complex object beam by solving a small system of equations; however, no noise model was considered.

Because of the drawbacks of existing approaches, we propose a new holographic reconstruction approach using a statistical technique [7]. Statistical image reconstruction for holography can be formulated as an inverse problem in which we try to obtain a complex reconstructed holographic image from hologram intensity data that are real. Cetin et al. [8] proposed a statistical technique for digital holography and other coherent imaging applications. However, their method was based on a simple image model and used a leastsquares approach.

According to the recording process of a hologram, our statistical model assumes a Poisson distribution having the mean associated with a squared magnitude of the interference between the object and reference beams. Due to the ill-posed nature of image reconstruction, our statistical technique uses penalized likelihood (PL) estimation. The likelihood function in this optimization problem contains multiple global minimizers. Therefore, regularization is necessary to improve the problem conditioning and to reduce nonuniqueness. Moreover, at least two measured holograms are needed to avoid an under-determined' problem when reconstructing a complex holographic image with the same number of pixels as the recorded hologram.

In PL estimation, the unknown parameter, which is the complex object wavefield, is estimated by minimizing a cost function. Since closed-form solutions are unavailable, we need an iterative algorithm to solve the problem. We approach this problem by using optimization transfer and convexity techniques. Instead of minimizing the original cost function, we minimize the surrogate function using an iterative algorithm, such as the separable-paraboloidal-surrogate $[9,10]$ or conjugate gradient algorithms. These methods monotonically decrease the cost function. 


\section{Measurement and Statistical Models}

Let $Y=\left[Y_{1}, \ldots, Y_{N}\right]$ denote the hologram measurement data recorded on a digital camera, where $N$ is the number of measurement elements. Because the measurement data are usually noisy, we consider the measurement reported by the $i$ th element of a digital camera to be a random variable whose mean is modeled as follows:

$$
\mathrm{E}\left[Y_{i}\right]=\left|[A x]_{i}+u_{i}\right|^{2}+b_{i}, \quad i=1, \ldots, N,
$$

where $A$ is the known system matrix, $x$ is the unknown parameters to be estimated, $u_{i}$ is the known reference beam, and $b_{i}$ is the known offset due to effects such as dark current. The goal is to estimate $x$ from the measured $Y_{i}$ 's, since $x$ parameterizes the unknown object of interest. The argument $[A x]_{i}$ belongs to the object beam which relates to the object through the system matrix. The system matrix $A$ can represent an imaging system matrix as well as Fresnel and Fourier transforms.

In statistical techniques for inverse problems, one uses the statistical characteristics of the measurement system to design the noise model. Since Poisson distributed photon noise is the dominant source of noise, we model the noisy measurement of the hologram recorded on a digital camera as a Poisson distribution with the mean described in (1):

$$
Y_{i} \sim \text { Poisson }\left\{[\mid A x]_{i}+\left.u_{i}\right|^{2}+b_{i}\right\}, \quad i=1, \ldots, N \text {. }
$$

Because the unknown image vector $x$ is complex, the size of the data vector $Y$ should be at least twice the number of elements of $x$, otherwise the problem will be under-determined.

To reconstruct a holographic image, we specify a cost function to be minimized. Since image reconstruction is an ill-posed problem, we focus on PL estimation having the cost function in the following form:

$$
\Phi(x)=L(x)+V(x)
$$

where $L$ denotes the negative log-likelihood function of the measurement, and $V$ denotes the roughness penalty function.

The negative log-likelihood function corresponding to the model (2) is given by:

$$
L(x)=\sum_{i=1}^{N} h_{i}\left([A x]_{i}\right)
$$

where the argument $l=l^{R}+\imath l^{I}$ for $h_{i}(l)$ is complex, and

$$
\begin{aligned}
h_{i}(l)= & h_{i}\left(l^{R}, l^{I}\right)=-y_{i} \log \left(\left|l+u_{i}\right|^{2}+b_{i}\right)+ \\
& \left(\left|l+u_{i}\right|^{2}+b_{i}\right)
\end{aligned}
$$

ignoring irrelevant constants independent of $x$. The superscripts $R$ and $I$ indicate the real and imaginary parts, respectively. The measured values $y_{i}$ 's that are real-valued are samples of independent Poisson random variables $Y_{i}$ 's.

We consider penalty functions that penalize the differences between neighboring object pixels using the following form $[10]$ :

$$
V(x)=\beta \sum_{i=1}^{r} \psi\left([C x]_{i}\right)
$$

where $\psi$ is a potential function, $C$ is a penalty matrix, $\beta$ is a regularization parameter that controls the degree of smoothness in the reconstructed image, and $r$ is the number of pairs of neighboring object pixels.

Our goal is to estimate $x$ by finding the minimizer of the cost function:

$$
\hat{x} \triangleq \arg \min _{x} \Phi(x) .
$$

Since closed-form solutions for the minimizer are unavailable, iterative algorithms are needed.

\section{The Algorithm}

Directly minimizing the cost function in (3) is diffcult when $h_{i}$ 's are nonquadratic. To simplify the optimization problem and to assure monotonic decreases in the cost function at each iteration, one can apply an optimization transfer approach by finding a "surrogate" function $\phi$ that lies above the cost function $[11,9,10]$. Therefore, we obtain the next estimate by minimizing the surrogate function instead:

$$
x^{n+1} \triangleq \arg \min _{x} \phi\left(x ; x^{n}\right)
$$

where $x^{n}$ denotes the estimate at the $n$th iteration.

We first focus on the likelihood part. Since quadratic choices for the surrogate $\phi$ are particularly easy to minimize, our goal now is to find a parabola that lies above the negative log-likelihood function. Figure 1 illustrates the one-dimensional plot of the marginal cost function $h_{i}\left(l^{R}, 0\right)$. In this plot, the marginal cost function has two optimal minima. However, a 2-D plot of $h_{i}\left(l^{R}, l^{I}\right)$ has multiple minimizers like a circle.

Because the negative log function is convex, we can apply De Pierro's multiplicative trick [12] to separate the real and imaginary parts of $h_{i}\left(l^{R}, l^{I}\right)$. Thus we obtain the following surrogate function:

$$
h_{i}\left(l^{R}, l^{I}\right) \leq h_{i}^{R}\left(l^{R} ; l^{n}\right)+h_{i}^{I}\left(l^{I} ; l^{n}\right)
$$




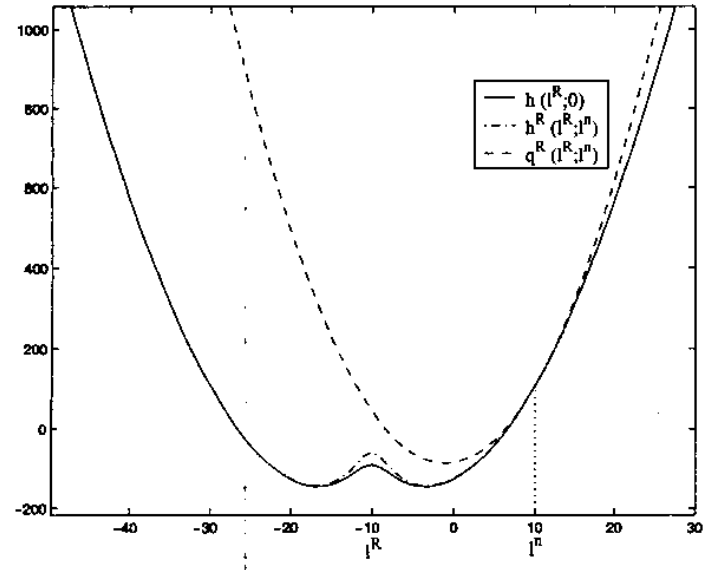

Figure 1: Illustration of the marginal cost, $h_{i}\left(l^{R}, 0\right)$, and surrogate functions as a function of $l^{R}$. The function with the dashed line is called the paraboloidal surrogate function which has the same first derivative and the same point as the original cost function at $l=l^{n}$.

where

$$
\begin{aligned}
h_{i}^{o}\left(l^{o} ; l^{n}\right) \triangleq & -y_{i} \alpha_{i}^{o, n} \log \left(\frac{\left(l^{o}+u_{i}^{o}\right)^{2}+b_{i} / 2}{\alpha_{i}^{o, n}}\right) \\
& +\left(l^{o}+u_{i}^{o}\right)^{2}+b_{i} / 2
\end{aligned}
$$

$\alpha_{i}^{o, n}=\frac{\left(l^{o, n}+u_{i}^{o}\right)^{2}+\dot{b}_{i} / 2}{k_{i}^{n}}, k_{i}^{n}=\left|l^{n}+u_{i}\right|^{2}+b_{i}, l^{n}=\left[A x^{n}\right]_{i}$, and $o$ represents the real or imaginary part.

The surrogates $h_{i}^{R}$ and $h_{i}^{I}$ each have two minima and are symmetric about the line $l=-u_{i}$. To facilitate the minimization in (8), we want to construct the following parabolic surrogate that lies above these curves:

$$
\begin{aligned}
q_{i}^{o}\left(l^{o} ; l^{n}\right)= & h_{i}^{o}\left(l^{o, n} ; l^{n}\right)+\dot{h}_{i}^{o}\left(l^{o, n} ; l^{n}\right)\left(l^{o}-l^{o, n}\right)+ \\
& \frac{1}{2} c_{i}^{o}\left(l^{o}-l^{o, n}\right)^{2}
\end{aligned}
$$

where $\dot{h}_{i}^{o}$ is the first derivative of $h_{i}^{o}$ and $c_{i}^{o}$ is the curvature of the parabola $q_{i}^{o}$. This parabola has the same value as $h_{i}^{\circ}$ at the current estimate $l^{\circ}=l^{o, n}$ and the same first derivatives at that point. To ensure that the parabolic surrogate lies above the original cost function [7], we have chosen the curvatures using the following general expression:

$$
c_{i}^{o}=\max _{l \in \mathbb{R}}\left\{\frac{\dot{h}_{i}^{o}\left(l ; l^{n}\right)-\dot{h}_{i}^{o}\left(l^{o, n} ; l^{n}\right)}{l-l^{o, n}}\right\} \text {. }
$$

Thus the likelihood surrogate function is

$Q\left(x ; x^{n}\right) \triangleq \sum_{i=1}^{N} q_{i}^{R}\left([A x]_{i}^{R} ;\left[A x^{n}\right]_{i}\right)+q_{i}^{I}\left([A x]_{i}^{I} ;\left[A x^{n}\right]_{i}\right)$.

Since this $Q$ function is quadratic, many algorithms could be applied to obtain the minimizer. In this paper, we use the separable-paraboloidal-surrogate (SPS) algorithm $[9,10]$ for this problem. The conjugate gradient (CG) method could also be applied easily because nonnegativity constraint is not enforced in this problem.

To apply the SPS approach, we separate pixels by using the additive convexity technique developed by De Pierro [12] so that simultaneous updating can be performed. The resulting function is called the separable paraboloidal surrogate function $Q_{j}$. Since $Q_{j}$ is a quadratic function of two variables: $x_{j}^{R}$ and $x_{j}^{I}$, minimizing $Q_{j}$ using Newton's method includes a $2 \times 2$ matrix-vector multiplication for each pixel as follows:

$$
\begin{aligned}
x_{j}^{n+1} & \triangleq \arg \min _{x_{j}} Q_{j}\left(x_{j}^{R}, x_{j}^{I} ; x^{n}\right) \\
& =x_{j}^{n}-H_{j}^{-1} \nabla Q_{j}\left(x_{j}^{n} ; x^{n}\right), \quad j=1, \ldots, P
\end{aligned}
$$

where the gradient of $Q_{j}$ and the $j$ th $2 \times 2$ Hessian matrix, $H_{j}$, are in (12) and (13).

Without regularization, a noisy image might be obtained after several iterations. Therefore, we derive the surrogate function for the penalty term in PL estimation. To be flexible, we separately penalize the real and imaginary parts. Thus, the penalty function can be expressed in the following form:

$$
V(x)=\beta^{R} \sum_{i=1}^{r} \psi\left(\left[C^{R} x^{R}\right]_{i}\right)+\beta^{I} \sum_{i=1}^{r} \psi\left(\left[C^{I} x^{I}\right]_{i}\right)
$$

where the superscripts $R$ and $I$ represent the real and imaginary parts. To preserve edges, we use a nonquadratic potential function $\psi$ of the following form [13]:

$$
\psi(t)=\delta^{2}\left[\left|\frac{t}{\tilde{\delta}}\right|-\log \left(1+\left|\frac{t}{\delta}\right|\right)\right]
$$

where $\delta$ is a user-specified parameter that controls the degree of edge preservation.

Similar to the nonquadratic likelihood function, we derive the surrogate functions, and the update of the SPS algorithm with regularization becomes

$$
\left(\begin{array}{c}
x_{j}^{R, n+1} \\
x_{j}^{J, n+1}
\end{array}\right)=\left(\begin{array}{c}
x_{j}^{R, n}-\frac{\left(d_{j}^{I I}+p_{j}^{R}\right)\left(\dot{L}_{j}^{R}+\dot{V}_{j}^{R}\right)-d_{j}^{R I}\left(\dot{L}_{j}^{I}+\dot{V}_{j}^{I}\right)}{\operatorname{det} \dot{H}_{\dot{j}}} \\
x_{j}^{I, n}-\frac{-d_{j}^{R I}\left(\dot{L}_{j}^{R}+\dot{V}_{j}^{R}\right)+\left(d_{j}^{R k}+p_{j}^{R}\right)\left(\dot{L}_{j}^{I}+\dot{V}_{j}^{I}\right)}{\operatorname{det} H_{j}}
\end{array}\right)
$$




$$
\begin{gathered}
\nabla Q_{j}\left(x_{j}^{n} ; x^{n}\right)=\left(\begin{array}{c}
\sum_{i=1}^{N} a_{i j}^{R} \dot{h}_{i}^{R}\left(\left[A x^{n}\right]_{i}^{R} ;\left[A x^{n}\right]_{i}\right)+a_{i j}^{I} \dot{h}_{i}^{I}\left(\left[A x^{n}\right]_{i}^{I} ;\left[A x^{n}\right]_{i}\right) \\
\sum_{i=1}^{N}-a_{i j}^{I} \dot{h}_{i}^{R}\left(\left[A x^{n}\right]_{i}^{R} ;\left[A x^{n}\right]_{i}\right)+a_{i j}^{R} \dot{h}_{i}^{I}\left(\left[A x^{n}\right]_{i}^{I} ;\left[A x^{n}\right]_{i}\right)
\end{array}\right)=\left(\begin{array}{c}
\frac{\partial L(x)}{\partial x_{j}^{R}} \\
\frac{\partial L x)}{\partial x_{j}^{I}}
\end{array}\right)_{x=x^{n}} \triangleq\left(\begin{array}{c}
\dot{L}_{j}^{R} \\
\dot{L}_{j}^{I}
\end{array}\right) \\
H_{j} \triangleq\left[\begin{array}{cc}
d_{j}^{R R} & d_{j}^{R I} \\
d_{j}^{I R} & d_{j}^{I I}
\end{array}\right]=\left[\begin{array}{cc}
\sum_{i=1}^{N} \frac{1}{\pi_{i j}}\left[\left(a_{i j}^{R}\right)^{2} c_{i}^{R}+\left(a_{i j}^{I}\right)^{2} c_{i}^{I}\right] & \sum_{i=1}^{N} \frac{a_{i j}^{R} a_{i j}^{I}}{\pi_{i j}}\left(-c_{i}^{R}+c_{i}^{I}\right) \\
\sum_{i=1}^{N} \frac{a_{j i j}^{R} a_{i j}^{I}}{\pi_{i j}}\left(-c_{i}^{R}+c_{i}^{I}\right) & \sum_{i=1}^{N} \frac{1}{\pi_{i j}}\left[\left(a_{i j}^{I}\right)^{2} c_{i}^{R}+\left(a_{i j}^{R}\right)^{2} c_{i}^{I}\right]
\end{array}\right]
\end{gathered}
$$

where $\dot{V}_{j}^{o}$ is the gradient of the penalty surrogate function, $p_{j}^{o, n}$ is the curvature of the penalty surrogate function, and $\tilde{H}_{j}$ is the new Hessian matrix

$$
\tilde{H}_{j}=H_{j}+\left[\begin{array}{cc}
p_{j}^{R} & 0 \\
0 & p_{j}^{I}
\end{array}\right] .
$$

\section{Simulation Results}

A $128 \times 128$ original image (Fig. $2 \mathrm{a}$ ) that is complex was degraded by the PSF, interference pattern, and Poisson noise (Fig. 2b) as in (2). We used a $7 \times 7$ jinc function, $\frac{J_{1}(2 \pi r)}{\pi r}$ where $J_{1}$ is a Bessel function of the first kind and $r$ is a polar-coordinate parameter, with full width at half maximum (FWHM) of 3.5 pixels as the PSF of the system, and the following reference beams:

$u_{r 1}\left(n_{1}, n_{2}\right) \Rightarrow 200 \exp \left(-\imath \frac{2 \pi}{3} n_{1}\right), n_{1}, n_{2}=0, \ldots, 127$

$u_{r 2}\left(n_{1}, n_{2}\right)=150 \exp \left(-\imath \frac{2 \pi}{4} n_{1}\right), n_{1}, n_{2}=0, \ldots, 127$.

The offset $b_{i}$ is assigned to be 5 and 10 for the first and second data, respectively. The Poisson noise has the peak signal-to-noise ratio (PSNR) of $29 \mathrm{~dB}$ and $24 \mathrm{~dB}$ in the first and second hologram data (Fig. 2b), respectively. The PSNR in the data is defined as follows:

$$
\mathrm{PSNR} \triangleq 10 \log _{10}\left[\frac{\max _{i}\left(y_{i}-b_{i}\right)^{2}}{\frac{1}{N} \sum_{i=1}^{N}\left(y_{i}-\mathrm{E}\left[y_{i}\right]\right)^{2}}\right] .
$$

Each simulated real-valued hologram data has the same size ( $128 \times 128$ pixels) as the original complexvalued image.

Figure 2c shows the conventional reconstruction using an apodizing Gaussian mask. The $41 \times 41$ Gaussian mask with FWHM of 27.2 pixels is performed on the selected region in the frequency domain of the hologram. The magnitude and phase of the reconstructed image appear to be blurry while noise still remains.
Owing to the effect of the filtering method, noise cannot be removed completely without oversmoothing edges. Figures $2 \mathrm{~d}$ shows our statistical holographic reconstruction. We used the image from the conventional approach as the initial image and included the nonquadratic penalty function with the regularization parameters $\beta^{R}=\beta^{I}=10$ and the edge-preserving parameters $\delta^{R}=\delta^{I}=1$. The SPS algorithm was run for 200 iterations. Unlike the conventional technique, the statistical technique with the nonquadratic penalty can greatly reduce noise while still preserving edges. The normalized root mean-squared error (NRMSE) in percentage is defined as follows:

$$
\text { NRMSE }=\frac{\left\|\hat{x}-x_{\text {true }}\right\|}{\left\|x_{\text {true }}\right\|} \times 100 \%
$$

where $\hat{x}$ is the reconstructed image, $x_{\text {true }}$ is the true image, and $\|\cdot\|$ represents the Euclidean norm.

Our statistical technique has a monotonic decreasing cost function. The unique global solution is not guaranteed in this problem because the original negative $\log$-likelihood function does not have a unique minimizer. Nevertheless, the penalty function can greatly reduce this non-uniqueness.

\section{Conclusions}

We have demonstrated the potential for reconstructing a digital holographic image using the proposed statistical technique. Because the method uses all the information in the recorded hologram rather than just one term, this approach can improve the quality of the image relative to the conventional numerical reconstruction technique that uses a spatial filter applied in the spatial frequency domain. Moreover, unlike the conventional approach, our statistical technique is not limited by the plane wave assumption of the reference beam. Because of the ill conditioning and non-uniqueness of the problem, our statistical holographic reconstruction is based on PL estimation. We constructed a statistical model for this system and developed a monotonic algorithm. Although a unique 

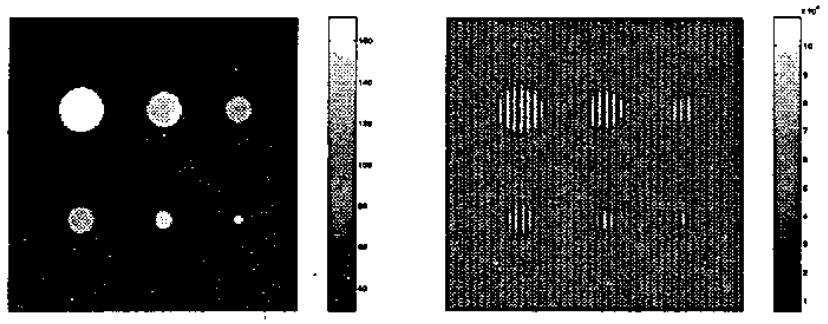

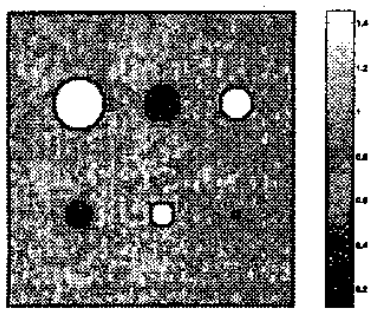

(a)
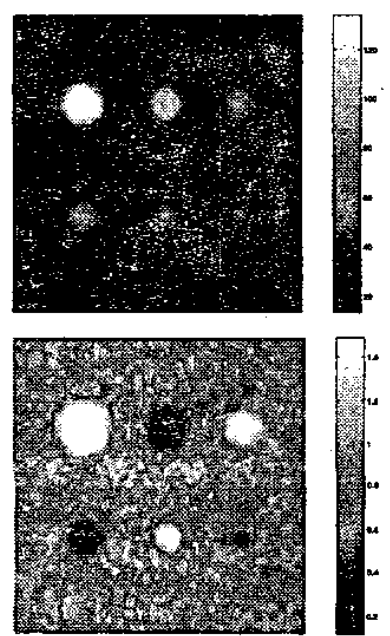

(c)

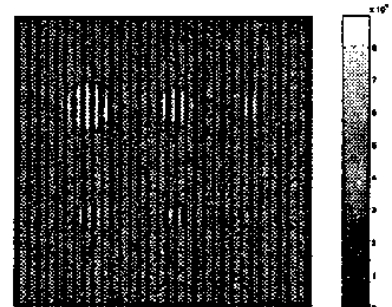

(b)
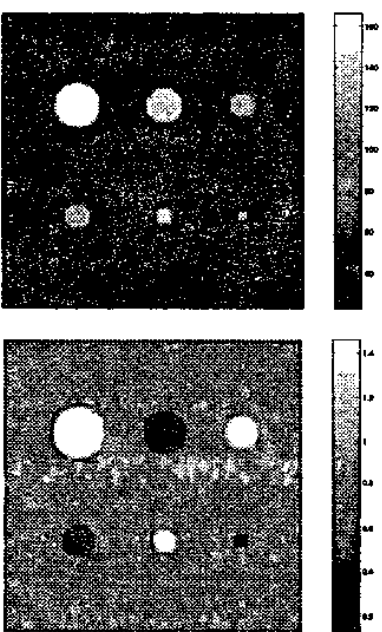

(d)
Figure 2: Holographic reconstruction of a complex object. The top image of each pair represents the magnitude and the bottom image represents the phase, except for the :hologram data. (a) Original image. (b) Two different hologram data. (c) Conventional reconstruction using an apodizing Gaussian filter (NRMSE $=40.0 \%$ ). (d) Statistical reconstruction using two data sets (NRMSE=14.1\%).

global minimum is not guaranteed due to the nonuniqueness nature of the negative log-likelihood function in this problem, we mostly overcome the problem of multiple minima through the help of the penalty function and by increasing the number of measure- ments.

\section{References}

[1] E. N. Leith and J. Upatnieks. Reconstructed Wavefronts and Communication Theory. J. Opt. Soc. Am. $A, 52(10): 1123-1130$, October 1962.

[2] E. Cuche, P. Marquet, and C. Depeursinge. Spatial filtering for zero-order and twin-image elimination in digital off-axis holography. Applied Optics, 39(23):4070-4075, August 2000.

[3] I. Yamaguchi and T. Zhang. Phase-shifting digital holography. Optics Letters, 22(16):1268-1270, August 1997.

[4] S. De Nicola, P. Ferraro, A. Finizio, and G. Pierattini. Wave front reconstruction of fresnel off-axis holograms with compensation of aberrations by means of phase-shifting digital holography. Optics and Lasers in Engineering, 37(4):331-340, April 2002.

[5] Y. Takaki, H. Kawai, and H. Ohzu. Hybrid holographic microscopy free of conjugate and zero-order images. Applied Optics, 38(23):4990-4996, August 1999.

[6] M. Liebling, T. Blu, E. Cuche, P. Marquet, C. Depeursinge, and $M$. Unser. A novel non-diffractive reconstruction method for digital holographic microcscopy. In Proc. IEEE Int'l Symp. on Biomedical Imaging, pages 625-628, 2002.

[7] S. Sotthivirat and J. A. Fessler. Statistical image reconstruction for digital holography. J. Opt. Soc. Am. $A, 2003$. Submitted.

[8] M. Çetin, W. C. Karl, and A. S. Willsky. Edgepreserving image reconstruction for coherent imaging applications. In Proc. IEEE Int'l Conf. on Image Processing, volume 2, pages 481-484, 2002.

[9] H. Erdoğan and J. A. Fessler. Monotonic Algorithms for Transmission Tomography. IEEE Trans. Med. Imaging, 18(9):801-814, September 1999.

[10] S. Sotthivirat and J. A. Fessler. Image Recovery Using Partitioned-Separable Paraboloidal Surrogate Coordinate Ascent Algorithms. IEEE Trans. Image Processing, 11(3):306-317, March 2002.

[11] J. A. Fessler. Grouped-coordinate ascent algorithms for penalized-likelihood transmission image reconstruction. IEEE Trans. Med. Imaging, 16(2):166-175, April 1997.

[12] A. R. De Pierro. A modified expectation maximization algorithm for penalized likelihood estimation in emission tomography. IEEE Trans. Med. Imaging, 14(1):132-137, March 1995.

[13] K. Lange. Convergence of EM Image Reconstruction Algorithms with Gibbs Smoothing. IEEE Trans. $M$. Imaging, 9(4):439-446, December 1990. 